\title{
Effect of Lonidamine on Systemic Therapy of DB-1 Human Melanoma Xenografts with Temozolomide
}

\author{
KAVINDRA NATH ${ }^{1}$, DAVID S. NELSON ${ }^{1}$, JEFFREY ROMAN ${ }^{1}$, MARY E. PUTT ${ }^{2}$, \\ SEUNG-CHEOL LEE ${ }^{1}$, DENNIS B. LEEPER ${ }^{3}$ and JERRY D. GLICKSON ${ }^{1}$ \\ Departments of ${ }^{1}$ Radiology and ${ }^{2}$ Biostatistics \& Epidemiology, \\ Perelman School of Medicine, University of Pennsylvania, Philadelphia, PA, U.S.A.; \\ ${ }^{3}$ Department of Radiation Oncology, Thomas Jefferson University, Philadelphia, PA, U.S.A.
}

\begin{abstract}
Background/Aim: Since temozolomide (TMZ) is activated under alkaline conditions, we expected lonidamine (LND) to have no effect or perhaps diminish its activity, but initial results suggest it may actually enhance either or both short- and long-term activity of TMZ in melanoma xenografts. Materials and Methods: Cohorts of 5 mice with subcutaneous xenografts $\sim 5 \mathrm{~mm}$ in diameter were treated with saline (control (CTRL)), LND only, TMZ only or LND followed by $T M Z$ at $t=40 \mathrm{~min}$ (time required for maximal tumor acidification). Results: Mean tumor volume for $L N D+T M Z$ for the period between 6 and 26 days was reduced compared to TMZ alone (repeated measures ANOVA $F(1,8), p=0.006)$, suggesting a pronounced impact of $L N D$ on this phenomenon. $T M Z$ and $L N D+T M Z$ produced median growth delays of 82 and 106 days, respectively. Conclusion: The use of TMZ alone and in combination with $L N D$ deserves further investigation in treatment of melanoma and other malignancies.
\end{abstract}

Melanoma, the most aggressive and life-threatening skin cancer, is the most rapidly increasing form of human cancer in the United States (1) and among Caucasian populations throughout the world (2). In the U.S., melanoma ranks fifth in incidence among males and seventh among females in 2016. Melanoma is not among the top ten causes of cancer death because surgical excision has proven successful in curing a large percentage of melanomas detected in early

This article is freely accessible online.

Correspondence to: Kavindra Nath, Ph.D., Perelman School of Medicine, Department of Radiology, Molecular Imaging Section, University of Pennsylvania, Philadelphia, 19104 PA, U.S.A. Tel: +1 2158981805, Fax: +1 2155732113, e-mail: kavindra.nath@ uphs.upenn.edu

Key Words: Melanoma, lonidamine, tumor acidification, tumor deenergization, temozolomide. stages. However, following recurrence with metastasis, the prognosis is poor. Mutationally-activated $B R A F$ is found in $40-60 \%$ of all melanomas with most common substitution of valine to glutamic acid at codon 600 (p. V600E) (3). Overall survival is approaching two years using agents that target this mutation (4, 5). MEK, RAS and other signal transduction inhibitors, in combination with mutant $B R A F$ inhibitors, have been used to deal with melanoma resistance to these agents (6). Treatment with anti-programmed death-1 (PD-1) checkpoint inhibitor immunotherapy currently produces durable response in about $25 \%$ of melanoma patients (7-10). Although these novel targeted therapies are promising, curative systemic treatment of melanoma remains elusive for the majority of melanoma patients. Based on various mechanisms of action, multiple therapeutic modalities can be developed for the treatment of melanoma. Here, we developed the potential to overcome melanomas being resistant to currently available treatments based on a single approach to increase melanoma-targeted specificity, drug delivery and minimize systemic toxicity. Furthermore, we introduced the use of lonidamine (LND)-induced potentiation of melanoma and other cancers to treatment with different nitrogen (N)-mustards and anthracyclines (11-15).

LND potentiates the activity of $\mathrm{N}$-mustards $(11,13-15)$. Nmustards alkylate guanine residues on DNA. This process is similar to the mechanisms of temozolomide (TMZ), and we have, thus, investigated the effect of LND on TMZ therapy in melanoma. TMZ is a second-generation alkylating agent undergoing clinical evaluation for patients with newly diagnosed, as well as recurrent malignant glioma and neuroendocrine tumors alone or in combination with radiotherapy (16). TMZ and dacarbazine (DTIC) are used to treat malignant melanoma and are clinically administered by oral and intravenous routes, respectively (17). These drugs are metabolized to the same active alkylating agents that methylate guanine residues of DNA at $\mathrm{N}^{7}(\sim 70 \%)$ and $\mathrm{O}^{6}(\sim 30 \%)$, with the latter alkylation site being more toxic to the tumor (18). Compared to DTIC, TMZ is well-tolerated and offers 
advantages in maintaining the quality of life of patients with metastatic melanoma and not requiring activation in the liver. Therefore, increasing the activities of TMZ by co-administration with LND may have considerable impact on clinical treatment of glioma, neuroendocrine tumors and a subset of melanomas.

\section{Materials and Methods}

Materials. LND was purchased from Santa Cruz Biotechnology, Inc. (Santa Cruz, CA, USA). LND was dissolved in a tris/glycine buffer $(22.0 \mathrm{mg} / \mathrm{ml})$ and administered intraperitoneally (i.p.) at a dose of $100 \mathrm{mg} / \mathrm{kg}$. The buffer used for dissolving LND consisted of trizma base $(2.4 \mathrm{~g})$ and glycine $(11.5 \mathrm{~g})$ in $100 \mathrm{ml}$ sterile water (final $\mathrm{pH}=8.3$ ). LND ( $5 \mathrm{mg}$ ) was dissolved in $227 \mu \mathrm{l}$ of buffer solution and vortexed until the solution was clear. TMZ, purchased from SigmaAldrich (St. Louis, MO, USA), was dissolved in DMSO and administered intravenously (i.v.).

Cell line. DB-1 melanoma cells were early passage human melanoma cells derived from a lymph node biopsy of a patient with metastatic disease that was excised before treatment and provided by Dr. David Berd (Thomas Jefferson University Hospital, Philadelphia, PA, USA). Cells were prepared from the tumor and cryopreserved after the $16^{\text {th }}$ passage. The presence of melanoma cell surface antigens was confirmed with monoclonal antibodies (19). DB-1 cells were grown as monolayers at $37^{\circ} \mathrm{C}$ in $5 \% \mathrm{CO}_{2}$ in $\alpha$-MEM (Invitrogen/Gibco, Carlsbad, CA, USA) supplemented with $10 \%$ fetal calf serum and $1 \%$ by volume with solutions of L-glutamine $(200 \mathrm{mM})$, HEPES $(1 \mathrm{M})$, penicillin-streptomycin $(10,000 \mathrm{U} / \mathrm{ml})$ and glucose $(2.5 \mathrm{M})$. The DB-1 cell line contained the following known pathogenic mutations: BRAF p.V600E; TP53 p.L145R; CDK4 p.R24C and TERT promoter region Chr5:1295228-1295229 GG>AA (15). Additional variants identified were PTEN p.T176A, JAK3 p.P693L, EGFR p.P753S and $A P C$ p.140G. The cell line also contained homozygous loss of $C D K N 2 B$ (15).

Animals and tumor cell implantation. Male nude mice purchased from the NIH at Charles River, Frederick, MD, USA, were housed in Micro-Isolator ${ }^{\circledR}$ cages with access to water and autoclaved mouse chow ad libitum. For tumor inoculation, animals were anesthetized with inhaled isoflurane (2\%). One million melanoma cells in $0.1 \mathrm{ml}$ of Hank's balanced salt solution (Invitrogen/Gibco) were inoculated subcutaneously above the right thigh of each animal. This regimen reliably produced $\sim 50 \mathrm{~mm}^{3}$ elliptical subcutaneous tumors approximately 5-6 weeks after inoculation.

Treatment with chemotherapy and tumor volume measurement. When tumors reached $\sim 50 \mathrm{~mm}^{3}$ in volume, four cohorts of male animals were randomized to the following treatment groups: cohort 1 (shamtreated CTRL) was infused i.v. with PBS and given appropriate sham i.p. injections of tris/glycine buffer; cohort 2 was infused i.v. with PBS $40 \mathrm{~min}$ after LND administration i.p. (100 mg/kg); cohort 3 was injected i.p. with tris/glycine buffer and infused i.v. with TMZ (18 $\mathrm{mg} / \mathrm{kg}$ delivered in $\sim 10 \mathrm{sec}$ ) in PBS; cohort 4 was injected i.p. with LND $(100 \mathrm{mg} / \mathrm{kg})$ and, after $40 \mathrm{~min}, \mathrm{TMZ}(18 \mathrm{mg} / \mathrm{kg})$ was infused i.v.

During the treatment and sham-treatment procedures, all animals were anesthetized with ketamine hydrochloride $(10 \mathrm{mg} / \mathrm{ml})$ and acepromazine maleate $(1 \mathrm{mg} / \mathrm{ml})$ with additional anesthesia being readministered as needed approximately every 45-60 min. Animals were placed on a water pad heater (Gaymar T-Pump, Kalamazoo, MI, USA) to maintain body temperature during anesthesia. Tail vein catheters (I.V. Catheters PTFE; Covidien, Mansfield, MA, USA) filled with heparin (100 USP Units/ml) to prevent blood clotting were placed using a restrainer (MTI Braintree Scientific, Braintree, MA, USA). TMZ was freshly prepared prior to injection. Depending on the treatment group, either tris/glycine or LND $(4.5 \mu \mathrm{l} / \mathrm{g})$ and TMZ or PBS $(5.0 \mu \mathrm{l} / \mathrm{g})$ were injected. Tail vein catheters were removed and animals were allowed to recover in cages.

For the first five days post-treatment, tumor volume and animal weight were measured daily with calipers (Scienceware, Bel-Art Products, Wayne, NJ, USA) and scale (Acculab PP401; H \& C Weighing Systems, Columbia, MD, USA), respectively. These measurements were repeated every two to three days up until 7 weeks and, then, with the exception of 15 weeks, weekly until 18 weeks. Animals were euthanized when their tumor volume reached $1.5 \mathrm{~cm}$ in longest diameter. Using the mean of the $\log$ of the volumes at 14 and 16 weeks, we interpolated the volume for five animals under observation at 15 weeks. The tumor dimensions were measured with a caliper in three orthogonal directions and the volume was calculated using the equation $\mathrm{V}=\pi(\mathrm{a} \times \mathrm{b} \times \mathrm{c}) / 6$, where $\mathrm{a}, \mathrm{b}$ and $\mathrm{c}$ are the length, width and depth of the tumor. All animal studies were performed in accordance with a protocol approved by the University of Pennsylvania Institutional Animal Care and Use Committee (IACUC).

Statistics. We explored the data graphically by fitting LOESSsmoothed curves to the tumor volume data. The primary outcome of interest was regrowth to two doublings of tumor volume at day 0 or, equivalently, four times the initial volume. TMZ in clinical practice is known to induce pseudo-progression; we observed transient increases in tumor volume followed by a decline within the first few weeks post-treatment. We defined this phenomenon as pseudoprogression. For the TMZ and LND+TMZ groups, pseudoprogression was observed in the first 3 to 5 weeks of the study; hence, for these animals we used first regrowth to two doublings after 3 weeks as the outcome. Because not all tumors exhibited regrowth, we used a Kaplan-Meier analysis to estimate the median time to regrowth and a log-rank test to compare failure times. Lower 95\% confidence intervals (CIs) are provided for the median; with a sample size of 5 per group, we were unable to determine the upper 95\% CI. Secondarily, we considered the effect of LND on TMZrelated pseudo-progression. We compared the mean relative tumor volume in the LND and the LND+TMZ groups using a repeated measures analysis of variance (ANOVA). The tumor volume data for each animal was normalized to day 0 . The day of measurement was included in the ANOVA model. Included in the results is the degrees of freedom for the relevant F-test along with the resulting p-value. The Type I error rate was set to 0.05 and all tests were two-sided. Analyses were conducted in R Studio (V 1.0.136) using R (Version 3.3.2; R Foundation for Statistical Computing, Vienna, Austria) (20).

\section{Results}

The mean tumor volume at the time of randomization was $46.0 \mathrm{~mm}^{3}$ (95\% CI=34.2-57.9), Figure 1 shows the relative tumor volume data for each animal. As expected, the CTRL and LND groups regrew rapidly. Regrowth in the TMZ and LND+TMZ groups was clearly delayed relative to the CTRL or LND groups, with 1 animal in each group not achieving the 

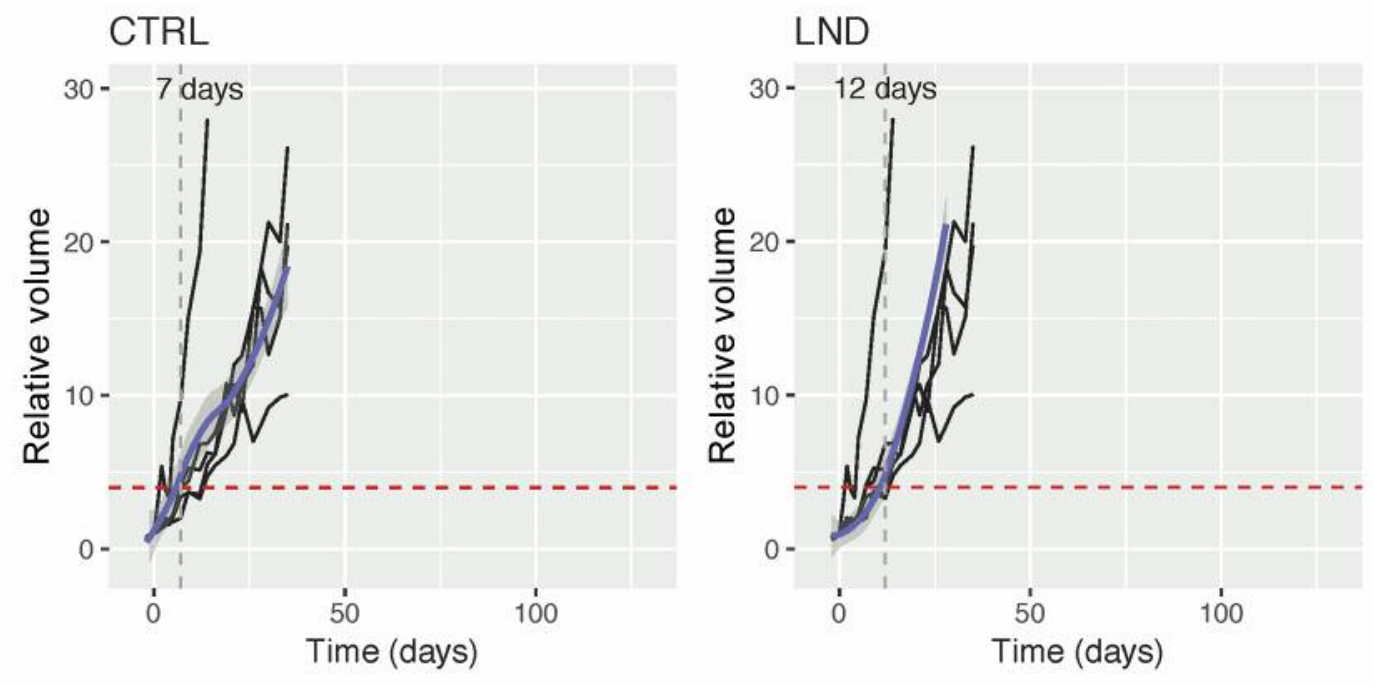

TMZ
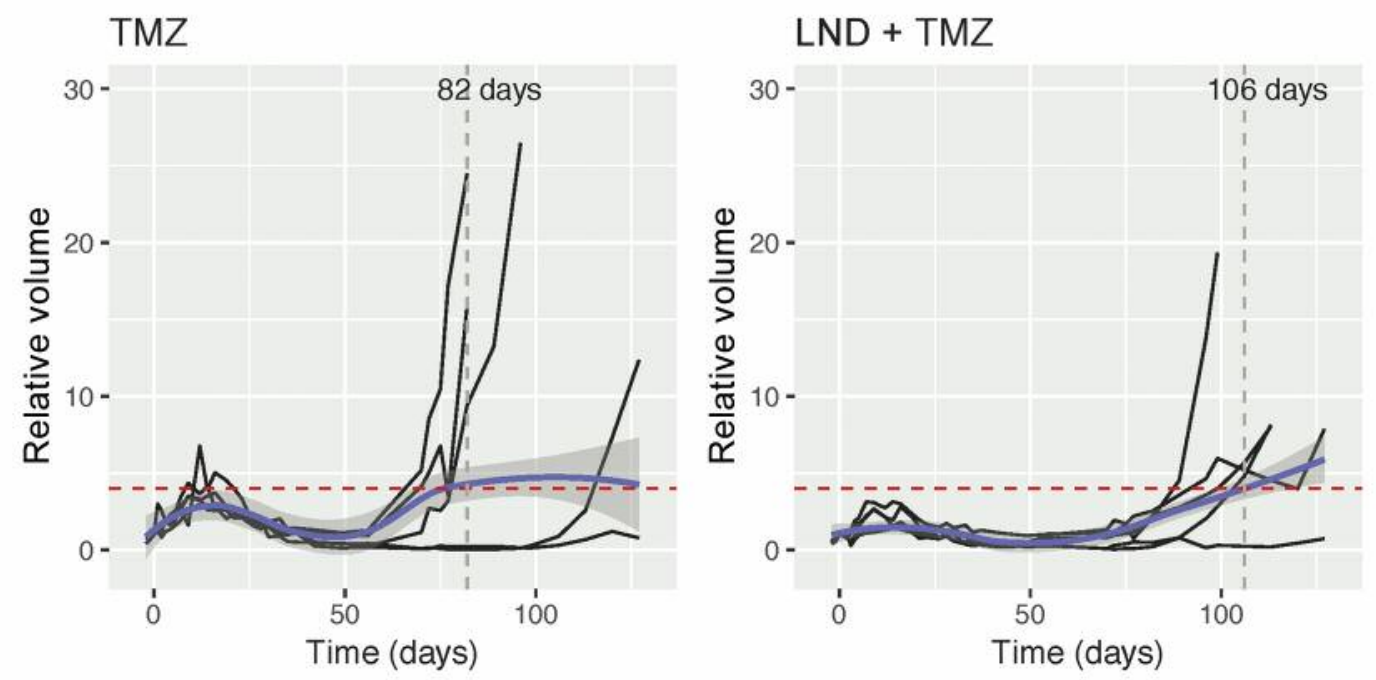

Figure 1. Growth delay experiments on DB-1 melanoma xenografts. Each panel shows the relative volume (normalized to day 0) for the individual animals. The blue line is the smoothed mean volume across all animals and the grey panel is the standard error of the fitted line. A value of two doublings relative to baseline (relative volume of 4 ) is indicated by the red dashed line. The median time to two doublings, as determined from the Kaplan-Meier method, is indicated by the vertical line and associated text. CTRL, Control; LND, lonidamine; TMZ, temozolomide.

endpoint of two doublings by the conclusion of the study. In addition, we note that both the TMZ and the TMZ+LND tumors exhibited pseudo-progression, defined here as an increase, followed by a decrease in volume in the initial period following treatment. Figure 2 shows a smaller scale version of the tumor volumes for the TMZ and LND+TMZ animals for the period prior to 40 days. Figure 3 shows the KaplanMeier plots for the experiment. Log-rank tests indicated that differences in time to regrowth between the groups were highly significant $(p<0.001)$. Notably, regrowth times for each of the LND+TMZ and the TMZ tumors differed from CTRL $(p=0.003)$. However, differences between LND+TMZ and TMZ were not statistically significant $(p=0.69)$.
Based on the F-test for a repeated measures ANOVA, differences between mean relative volumes for TMZ and LND+TMZ for the period between 6 and 26 days were highly significant (repeated measures ANOVA F $(1,8), p=0.006)$. For TMZ during this period, the mean volume relative to the day 0 was 3.0 (95\% $\mathrm{CI}=2.3-3.9)$; for $\mathrm{LND}+\mathrm{TMZ}$ it was 1.6 (95\% CI=1.2-2.2). The median time (with a 95\% lower confidence limit) to two doublings appears in Table I. Kaplan-Meier estimates of median time to regrowth (two doublings) ranged from 7 (CTRL) to 12 days (LND) in the CTRL group and were 82 days in the TMZ group and 106 days in the LND+TMZ group. Differences in time to regrowth between treatment groups were highly significant 

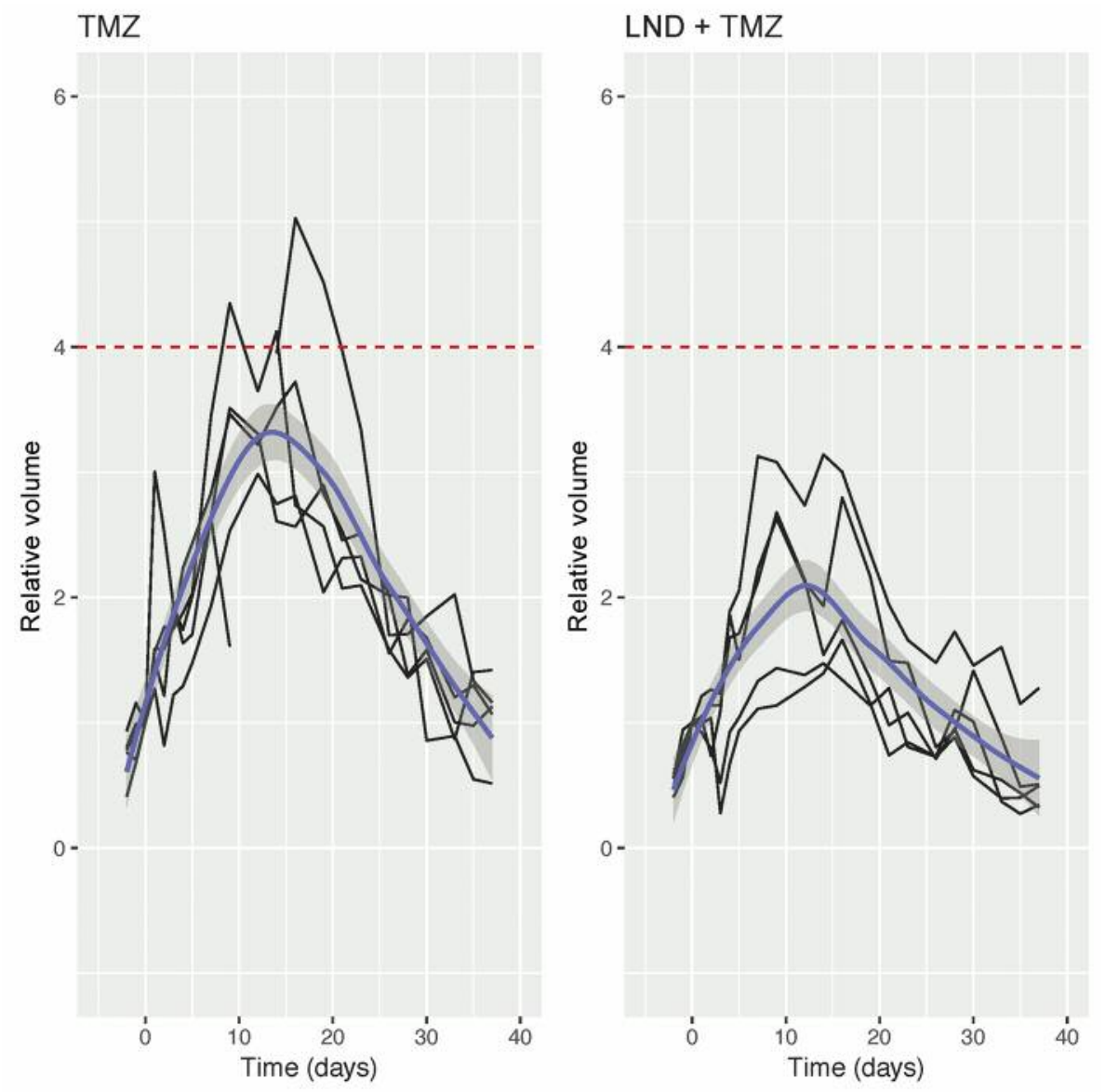

Figure 2. Pseudo-progression in the TMZ and LND+TMZ animals. The data are the same as in Figure 1 but the time scale is less than 40 days in order to visualize pseudo-progression. Blue line is the LOESS-smoothed mean. Grey bars are the standard error of the mean. The red dashed line shows two doublings. LND, Lonidamine; TMZ, temozolomide.

(log-rank test, $p<0.001)$ with TMZ and LND+TMZ each differing from CRTL and LND ( $p=0.003$ for each pairwise comparison). Differences in regrowth between TMZ and LND+TMZ were not statistically significant $(p=0.69)$.

We have presented ${ }^{31} \mathrm{P}$ and ${ }^{1} \mathrm{H}$ magnetic resonance spectroscopy (MRS) results (Figure 4) in the current study with representative phosphorus spectra to delineate the time window for intracellular acidification, bioenergetic decline and lactate production in DB-1 melanoma xenografts following LND administration at time zero $(11,14)$. In the current study, TMZ was injected at $\mathrm{t}=40 \mathrm{~min}$ after LND administration.

\section{Discussion}

Our recent ${ }^{31} \mathrm{P}$ and ${ }^{1} \mathrm{H}$ MRS studies have shown that treatment of DB-1 melanoma xenografts with LND (100 mg/kg, i.p.) selectively lowers the intracellular $\mathrm{pH}(\mathrm{pHi})$ of tumors from $6.90 \pm 0.05$ to $6.33 \pm 0.1$ with a minimal decrease of the
Table I. Kaplan-Meier-based estimates of time to regrowth (2 doublings) for each treatment group.

\begin{tabular}{lc}
\hline Treatment group & Median time to regrowth (days) $(95 \% \mathrm{CI})$ \\
\hline CTRL & $7(7, \mathrm{NA})$ \\
LND & $12(9, \mathrm{NA})$ \\
TMZ & $82(70, \mathrm{NA})$ \\
LND+TMZ & $106(96, \mathrm{NA})$ \\
\hline
\end{tabular}

CTRL, Control; LND, lonidamine; TMZ, temozolomide; CI, confidence interval; NA, not available.

extracellular $\mathrm{pH}$ from $7.00 \pm 0.04$ to $6.80 \pm 0.07$. The energy state of the tumor that is measured by the nucleoside triphosphate/inorganic phosphate $(\mathrm{NTP} / \mathrm{Pi})$ ratio decreased monotonically reaching $66.8 \pm 5.7 \%$ of its pretreatment level in $3 \mathrm{~h}$. Although initial investigators of LND activity claimed 
Tumor regrowth (two doublings)

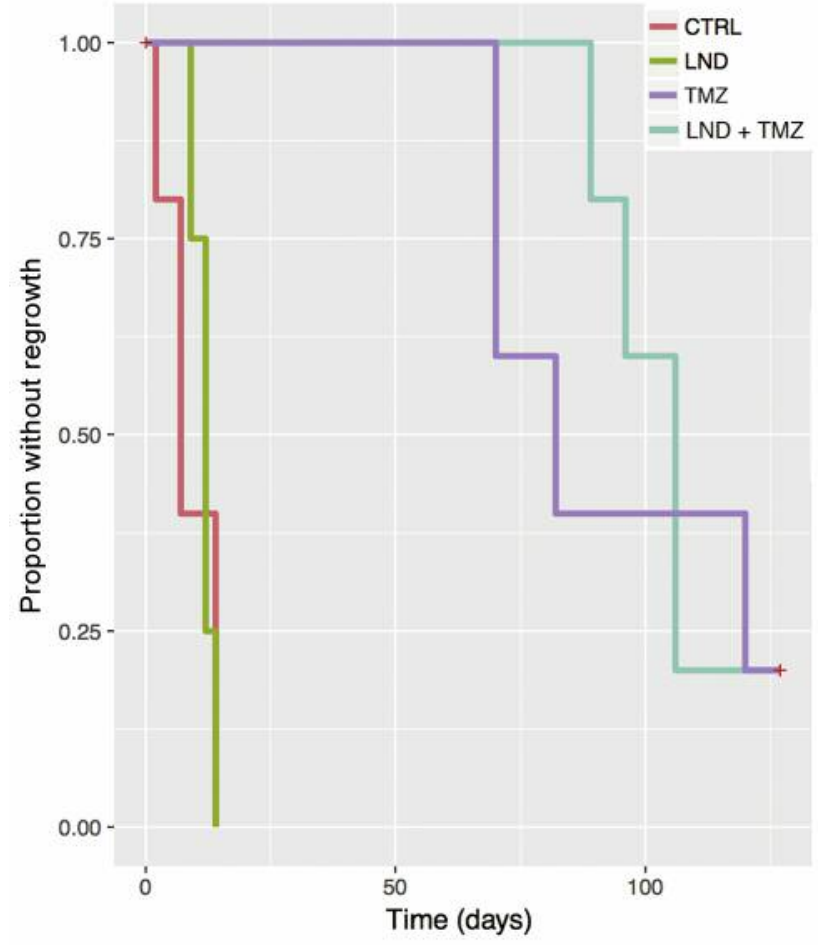

Figure 3. Kaplan-Meier plot of regrowth to two doublings. CTRL, Control; LND, lonidamine; TMZ, temozolomide.

that this agent inhibited glycolytic activity at hexokinase II (21), we observed that the level of lactic acid in the tumor increased 3-fold over $3 \mathrm{~h}(11,13,14)$. Tumor acidification and de-energization were detected in a variety of tumors grown as xenografts in mice, including various melanomas, both triplypositive and triply-negative breast tumors, ovarian cancer and prostate cancer (12). At this dose level, LND produced no effect on normal skeletal muscle or brain and had only a small transient effect on liver metabolism $(13,14)$.

The Warburg effect discovered by Otto Warburg states that most tumors exhibit high level of aerobic glycolysis and has been exploited for the detection and treatment of cancer (22). We wanted to exploit this natural preference of tumors for glycolytic metabolism as a method to selectively induce intracellular acidification of the tumor by accumulation of lactic acid even under aerobic conditions and use this selective tumor acidification and de-energization to enhance tumor response to chemotherapeutic agents, such as $\mathrm{N}$-mustards and doxorubicin (11-15). Others had shown that LND sensitizes tumors to hyperthermia $(23-26)$, radiotherapy $(27,28)$ and photodynamic therapy (29) by a variety of different mechanisms. In addition, to selectively acidifying and deenergizing human melanoma xenografts, LND had similar effects on mouse xenografts of triply-negative and triplypositive human breast cancer, human ovarian cancer and human prostate cancer (12), with the effect of LND increasing with the extent of glycolytic metabolism in the tumor.

LND, first introduced in 1979 as an anti-spermatogenic agent, (30) has limited antineoplastic activity as a single agent but has exceptional potential in modulating the activities of conventional cancer therapeutic agents. Its mechanism of action has been the subject of controversy with initial reports by Floridi et al. $(21,31,32)$ claiming that it inhibited glycolysis at the level of hexokinase II, which they believed explained the decreased secretion of lactic acid by Ehrlich ascites tumor cells. Floridi then collaborated with Lehninger (33) and others (34) to demonstrate that LND inhibited tumor respiration at the level of the mitochondrial electron transport chain but did not unambiguously delineate the specific site of inhibition. Ben-Horin et al. (35) and Ben-Yoseph et al. (36) then performed multinuclear MRS studies on perfused breast cancer cells and 9L glioma cells in culture and in vivo as subcutaneous tumors in rats. These studies, as well as subsequent ${ }^{13} \mathrm{C}$ MRS studies using diffusion-weighted spectroscopy to distinguish cell metabolites from metabolites in the perfusate of a bioreactor containing MCF-1 breast cancer cells by Mardor et al. (37), demonstrated that lactic acid was accumulating in the tumor cytosol, which they attributed to inhibition of export of lactic acid via the monocarboxylic acid transporter(s) (MCTs). Our recent publications $(11,38)$ confirmed that LND inhibits lactate export by the proton-linked MCTs and specifically measured inhibition by MCT1and MCT4 that commonly are expressed in many tumor types; however, our studies demonstrated that an even more potent inhibition of pyruvate export from the cytosol into the mitochondria via the mitochondrial pyruvate carrier (MPC) was augmenting cytosolic acidification and also explained a marked decrease in tumor ATP production by oxidative phosphorylation. This inhibition of mitochondrial respiration was further increased at higher LND concentration by inhibition of electron-transport at Complex II at the ubiquinone reductase step $(11,39)$. Our studies, therefore, confirm inhibition of the MCTs but indicate that inhibition of the MPC may play an important role in retention of lactic acid and pyruvate in the cytosol. Inhibition of both the MPC and Complex II explain the effect of LND on tumor respiration that produces the selective effect of this drug on tumor energy status. Selective tumor acidification enhances the activities of nitrogen mustard alkylating agents by a complex mechanism that has been described extensively (40-46) that involves stabilizing the alkylating intermediate aziridinium cation, which is degraded by $\mathrm{OH}^{-}$ions; inhibition of glutathione transferase activity also neutralizes aziridinium cation intermediates and acid inhibition of $\mathrm{O}^{6}$-alkyltransferase also called $\mathrm{O}^{6}$-methylguanine transferase (MGMT), a key enzyme involved in 

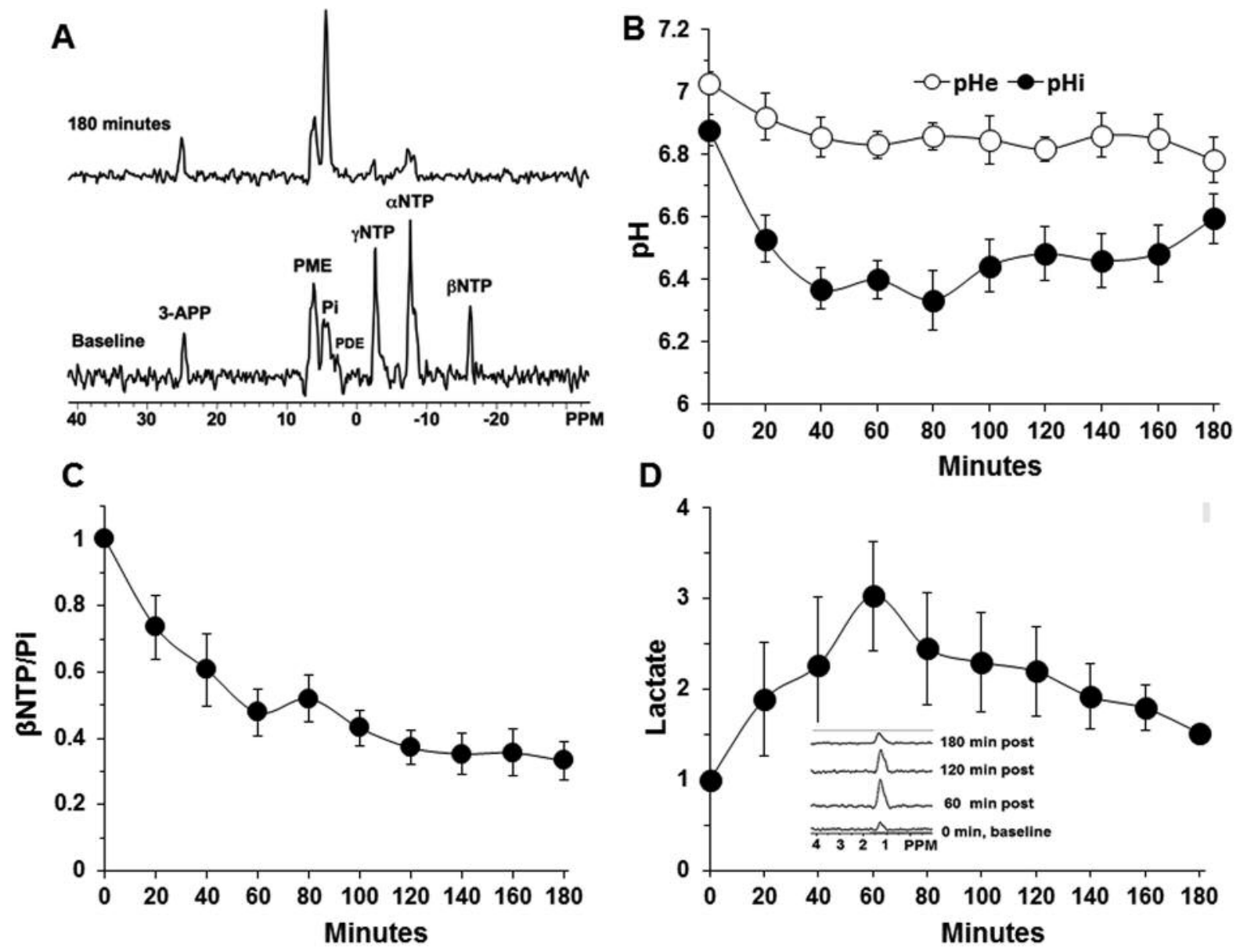

Figure 4. The intracellular $\mathrm{pH}(\mathrm{pHi})$, extracellular $\mathrm{pH}(\mathrm{pHe})$, bioenergetics and lactate profile of human melanoma xenograft after lonidamine (LND) administration. (A) In vivo localized (Image Selected In vivo Spectroscopy-ISIS) 31-Phosphorus magnetic resonance spectroscopy ( ${ }^{31} P$ MRS) spectra of a human melanoma xenograft grown subcutaneously in nude mice (lower) pre-and (upper) 180 min post-administration of LND (100 mg/kg, i.p.). Resonance assignments are as follows, 3-aminopropylphosphonate (3-APP); phosphomonoesters (PME); inorganic phosphate (Pi); phosphodiesters $(P D E) ; \gamma$ nucleoside-triphosphate ( $\gamma N T P), \alpha$ nucleoside-triphosphate $(\alpha N T P), \beta$ nucleoside-triphosphate $(\beta N T P)$. Decrease in $\beta N T P$ levels and the corresponding increase in Pi following LND administration (Upper spectrum of panel A) are indicating impaired energy metabolism. (B) pHi, pHe profile as a function of time. (C) The changes of bioenergetics ( $\beta N T P / P i)$ (ratio of peak area) relative to baseline. $(D)$ Change in tumor lactate as a function of time, inset picture showing lactate spectra using ${ }^{1} \mathrm{H}$ MRS with Hadamard Selective Multi Quantum Coherence transfer pulse sequence in human melanoma xenografts. Area under the curve was compared to baseline at each time point and was normalized to baseline levels as a function of time in response to LND $(100 \mathrm{mg} / \mathrm{kg} ;$ i.p. $)$ administered at time zero. The values are presented as mean \pm S.E.M. When not displayed, S.E.M. values were smaller than the symbol size. This figure was originally published in our previous publication (BBA Reviews in Cancer (11)) and presented here again in the current manuscript to better delineate the time window of intracellular acidification, bioenergetics decline and lactate production in DB-1 melanoma xenografts following LND administration at time zero.

repair $\mathrm{N}$-mustard alkylation of DNA at the $\mathrm{N}^{7}$ and $\mathrm{O}^{6}$ positions of guanine, also contributes to acid potentiation of $\mathrm{N}$-mustard antineoplastic activity. Potentiation of doxorubicin activity appears to occur by a cation-trapping mechanism; the neutral weakly basic form of doxorubicin crosses the plasma membrane and is trapped in the tumor cell as a result of protonation in the acidified cytosol. In addition, both $\mathrm{N}$ mustards like melphalan, as well as doxorubicin, are subject to multi-drug resistance mediated by the p-glycoprotein that pumps these cytotoxic drugs out of the tumor cell by an energy-dependent mechanism, which is potentially diminished by LND-mediated tumor de-energization. Thus, the mechanism of LND activity as delineated by Floridi et al. $(33,34)$ Ben-Horin et al. (35) Ben-Yoseph et al. (36) and Mardor et al. (37) and refined and extended by our laboratory $(11-15,38,39)$ explains the ability of LND to enhance the activity of two important classes of antineoplastic agents, the $\mathrm{N}$-mustards and anthracyclines. 
The goal of the present study was to determine how intracellular tumor acidification and de-energization induced by LND might influence the efficacy of TMZ in DB-1 melanoma xenografts. Although $\mathrm{N}$-mustards have been used in the clinical treatment of melanoma and soft-tissue sarcomas in the setting of hyperthermic isolated limb perfusion $(47,48)$, these procedures are no longer commonly performed. However, TMZ has been used in the treatment of melanoma $(17,49,50)$ and is the standard of practice (SOP) in the treatment of gliomas (51-54) and neuro-endocrine tumors (55-59). DTIC has been until recently the standard therapy of melanoma but recently replaced by targeted kinase therapy directed against mutant $B R A F$ and $M E K$ signaling pathways, as well as immune checkpoint inhibitors $(3,6)$.

Denny et al. (18) report that TMZ is stable in acid but decomposes to mitozolomide (MTIC) by a base-catalyzed ring-opening reaction involving activated water molecules and followed by additional steps leading to formation of methyldiazonium cation, the reactive electrophilic species that alkylates guanine at $\mathrm{N}^{7}$ and $\mathrm{O}^{6}$, at approximately a 70:30 ratio, with the $\mathrm{O}^{6}$ alkylated species being more toxic. The cancer drug DTIC is also metabolized to MTIC by reaction with liver oxygenases; hence, DTIC is best administered by i.v. injection, whereas $\mathrm{TMZ}$ can be administered orally; however, both drugs can produce similar DNA alkylation products with different pharmacokinetic characteristics. Because TMZ is converted to MTIC under mildly alkaline conditions, we expected that it would not be activated by LND, which selectively acidifies tumors. We, therefore, expected it to be a negative CTRL in studies of agents that are potentiated by LND; we found that LND may potentiate the activity of TMZ producing a much more potent overall effect than we had encountered with nitrogen mustards (15). The mechanism of acid-catalyzed alkylation of guanine by TMZ has not been clearly delineated. It appears, however, to involve conversion of TMZ to MTIC, which then is converted to diazomethane and the methyldiazonium cation $(18,60)$. The methyldiazonium ion is a potent electrophilic alkylating agent that binds in the major groove of DNA interacting preferentially with runs of multiple guanine residues and alkylating this base at $\mathrm{O}^{6}$ or $\mathrm{N}^{7}$. The methylated guanine pairs preferentially with thymine instead of guanine causing misreading of DNA and RNA and effectively producing an apparent $\mathrm{C} \rightarrow \mathrm{T}$ mutation.

A number of repair mechanisms act to correct this coding error. The enzyme MGMT removes the alkylating group from guanine, thereby correcting the mutation and reversing the activity of TMZ (61). Alternatively, the mismatch repair enzymes (MMR) excise the erroneous $\mathrm{T}$ base. However, unless the $\mathrm{O}^{6}$-methylG:T mismatch is repaired before the resynthesis step in the MMR pathway occurs, thymine is likely to be re-inserted, leading to a futile cycle of repetitive excisions that eventually result in a single-strand break and apoptosis. Double-stranded breaks also occur after multiple steps of futile repair cycling. Of the five proteins involved in the MMR pathways, two appear to play a critical role in TMZ response: MLH-1 and MSH-2. High levels of these proteins and low or non-existent levels of MGMT are required for maximal TMZ response, whereas the reverse situation favors resistance to this drug. These agents serve as biomarkers and predictors of therapeutic response by TMZ.

Middlemas et al. (61) have examined xenografts derived from 17 pediatric solid tumors (mostly neuroblastomas and soft-tissue sarcomas). TMZ was administered by oral gavage at $66 \mathrm{mg} / \mathrm{kg}$ for five consecutive days and the cycle was repeated every 21 days for 3 cycles. Five lines exhibited complete responses (disappearance of the tumor for some time within the 12 week cycle) and three more lines showed partial response ( $>50$ volume regression) corresponding to an overall response rate of $47 \%$. For the DB-1 melanoma xenografts, we also observed a complete response (disappearance of the tumor within 12 weeks) in our TMZ and TMZ+LND treated animals (i.e., apparent cures) with a single i.v. dose of TMZ $(18 \mathrm{mg} / \mathrm{kg})$ or a single dose of LND (100 mg/kg, i.p.) followed by the same dose of TMZ 40 min later (to permit maximal tumor acidification). We surmise that the DB-1 tumor was highly responsive and probably expressed low or non-existent levels of MGMT and high levels of MLH-1 and MSH-2. As with melphalan and doxorubicin, LND may further enhance the activity of TMZ by diminishing the bioenergetics state of the tumor (i.e., ATP levels), thereby inhibiting multidrug resistant export of TMZ.

Oral administration of TMZ used to be the SOP for treatment of advanced disseminated melanoma (17, 49, 50); subsequent studies demonstrated that DTIC, when administered i.v., was equivalent to TMZ although TMZ is still considered superior to dacarbazine at maintaining or improving physical function and avoiding fatigue and insomnia. As noted above, both TMZ and DTIC have been displaced from the treatment of melanoma by targeted kinase inhibitors and immune checkpoint inhibitors but neither of these methods is curative; about half the melanoma patients do not express the BRAF mutation that is targeted in the former treatment and about $75 \%$ of the melanoma patients fail to experience a durable response from immunotherapy. There appears to be a subset of melanoma patients who may benefit from treatment with TMZ perhaps administered together with LND and there are biomarkers, low levels of MGMT and high levels of MMR proteins that could identify these patients. These considerations point to the need for further development of TMZ+LND for the treatment of these patients. These methods will also benefit patients with gliomas and neuroendocrine tumors for whom TMZ remains an acknowledged standard therapy. 


\section{Acknowledgements}

Study was supported by NIH grants R01-CA1 129544, R01CA172820 and utilized the facilities of the Small Animal Imaging Facility of the University of Pennsylvania. We thank Dr. Marco G. Paggi, Regina Elena National Cancer Institute, Rome, Italy for critical comments. Kevin Muriuki and Stepan Orlovskiy are acknowledged for their help to perform this study.

\section{References}

1 American Cancer Society: Cancer facts and figures 2017.

2 Maclennan R, Green AC, McLeod GRC and Martin NG: Increasing incidence of cutaneous melanoma in Queensland, Australia. J Nat Cancer Inst 84: 1427-1432, 1992.

3 Flaherty KT, Puzanov I, Kim KB, Ribas A, McArthur GA, Sosman JA, O'Dwyer PJ, Lee RJ, Grippo JF, Nolop K and Chapman PB: Inhibition of mutated, activated BRAF in metastatic melanoma. N Engl J Med 363: 809-819, 2010.

4 Ascierto PA, McArthur GA, Dreno B, Atkinson V, Liszkay G, Di Giacomo AM, Mandala M, Demidov L, Stroyakovskiy D, Thomas L, de la Cruz-Merino L, Dutriaux C, Garbe C, Yan Y, Wongchenko M, Chang I, Hsu JJ, Koralek DO, Rooney I, Ribas $\mathrm{A}$ and Larkin J: Cobimetinib combined with vemurafenib in advanced BRAF(V600)-mutant melanoma (coBRIM): Updated efficacy results from a randomised, double-blind, phase 3 trial. Lancet Oncol 17: 1248-1260, 2016.

5 Larkin J, Ascierto PA, Dreno B, Atkinson V, Liszkay G, Maio M, Mandala M, Demidov L, Stroyakovskiy D, Thomas L, de la Cruz-Merino L, Dutriaux C, Garbe C, Sovak MA, Chang I, Choong N, Hack SP, McArthur GA and Ribas A: Combined vemurafenib and cobimetinib in BRAF-mutated melanoma. $\mathrm{N}$ Engl J Med 371: 1867-1876, 2014.

6 Long GV, Stroyakovskiy D, Gogas H, Levchenko E, de Braud F, Larkin J, Garbe C, Jouary T, Hauschild A, Grob JJ, Sileni VC, Lebbe C, Mandala M, Millward M, Arance A, Bondarenko I, Haanen J, Hansson J, Utikal J, Ferraresi V, Kovalenko N, Mohr P, Probachai V, Schadendorf D, Nathan P, Robert C, Ribas A, DeMarini DJ, Irani JG, Casey M, Ouellet D, Martin AM, Le N, Patel $\mathrm{K}$ and Flaherty K: Combined BRAF and MEK inhibition versus BRAF inhibition alone in melanoma. N Eng J Med 371: 1877-1888, 2014.

7 Antonia SJ, Larkin $\mathrm{J}$ and Ascierto PA: Immuno-oncology combinations: A review of clinical experience and future prospects. Clin Cancer Res 20: 6258-6268, 2014.

8 Mahoney KM, Freeman GJ and McDermott DF: The next immune-checkpoint inhibitors: PD-1/PD-L1 blockade in melanoma. Clin Therapeutics 37: 764-782, 2015.

9 Postow MA, Callahan MK and Wolchok JD: Immune checkpoint blockade in cancer therapy. J Clin Oncol 33: 1974-U1161, 2015.

10 Shin DS and Ribas A: The evolution of checkpoint blockade as a cancer therapy: What's here, what's next? Current Opinion Immunol 33: 23-35, 2015.

11 Nath K, Guo L, Nancolas B, Nelson DS, Shestov AA, Lee SC, Roman J, Zhou R, Leeper DB, Halestrap AP, Blair IA and Glickson JD: Mechanism of antineoplastic activity of lonidamine. Biochim Biophys Acta 1866: 151-162, 2016.

12 Nath K, Nelson DS, Heitjan DF, Leeper DB, Zhou R and Glickson JD: Lonidamine induces intracellular tumor acidification and ATP depletion in breast, prostate and ovarian cancer xenografts and potentiates response to doxorubicin. NMR Biomed 28: 281-290, 2015.

13 Nath K, Nelson DS, Heitjan DF, Leeper DB, Zhou R and Glickson JD: Effects of hyperglycemia on lonidamine-induced acidification and de-energization of human melanoma xenografts and sensitization to melphalan. NMR Biomed 28: 395-403, 2015.

14 Nath K, Nelson DS, Ho AM, Lee SC, Darpolor MM, Pickup S, Zhou R, Heitjan DF, Leeper DB and Glickson JD: ${ }^{31} \mathrm{P}$ and ${ }^{1} \mathrm{H}$ MRS of DB-1 melanoma xenografts: Lonidamine selectively decreases tumor intracellular $\mathrm{pH}$ and energy status and sensitizes tumors to melphalan. NMR Biomed 26: 98-105, 2013.

15 Nath K, Nelson DS, Putt ME, Leeper DB, Garman B, Nathanson $\mathrm{KL}$ and Glickson JD: Comparison of the lonidamine potentiated effect of nitrogen mustard alkylating agents on the systemic treatment of DB-1 human melanoma xenografts in mice. Plos One 11(6): e0157125, 2016.

16 Tatar Z, Thivat E, Planchat E, Gimbergues P, Gadea E, Abrial C and Durando X: Temozolomide and unusual indications: Review of literature. Cancer Treat Rev 39: 125-135, 2013.

17 Li RH, Hou XY, Yang CS, Liu WL, Tang JQ, Liu YQ and Jiang G: Temozolomide for treating malignant melanoma. J Coll Physicians Surg Pak 25: 680-688, 2015.

18 Denny BJ, Wheelhouse RT, Stevens MFG, Tsang LLH and Slack JA: NMR and molecular modeling investigation of the mechanism of activation of the antitumor drug temozolomide and its interaction with DNA. Biochemistry 33: 9045-9051, 1994.

19 Hill LL, Korngold R, Jaworsky C, Murphy G, McCue P and Berd D: Growth and metastasis of fresh human melanoma tissue in mice with severe combined immunodeficiency. Cancer Res 51: 4937-4941, 1991.

20 Efron TB: Bootstrap Method for standard errors, confidence intervals and other measures of statistical accuracy. Statistical Sci 1: 54-77, 1986.

21 Floridi A, Paggi MG and Fanciulli M: Modulation of glycolysis in neuroepithelial tumors. J Neurosurg Sci 33: 55-64, 1989.

22 Warburg O: On the origin of cancer cells. Science (New York, NY) 123: 309-314, 1956.

$23 \mathrm{Kim} \mathrm{JH,} \mathrm{Kim} \mathrm{SH,} \mathrm{Alfieri} \mathrm{A,} \mathrm{Young} \mathrm{CW} \mathrm{and} \mathrm{Silvestrini} \mathrm{B:}$ Lonidamine - A hyperthermic sensitizer of hela cells in culture and of the Meth-A tumor in vivo. Oncology 41: 30-35, 1984.

24 Ning SC and Hahn GM: Combination therapy: Lonidamine, hyperthermia and chemotherapy against the RIF-1 tumor in vivo. Cancer Res 51: 5910-5914, 1991.

25 Silvestrini B, Hahn GM, Cioli V and Demartino C: Effects of lonidamine alone or combined with hyperthermia in some experimental cell and tumor systems. Br J Cancer 47: 221-231, 1983.

26 Teicher BA, Holden SA, Ara G and Menon K: Whole-body hyperthermia and lonidamine as adjuvant therapy to treatment with cisplatin with or without local radiation in mouse bearing the Lewis lung carcinoma. Int J Hyperthermia 11: 637-645, 1995.

$27 \mathrm{Kim} \mathrm{JH}$, Alfieri A, Kim SH, Young CW and Silvestrini B: Radiosensitization of Meth-A fibrosarcoma in mice by lonidamine. Oncology 41: 36-38, 1984.

$28 \mathrm{Kim} \mathrm{JH}$, Alfieri AA, Kim SH and Young CW: Potentiation of radiation effects on two murine tumors by lonidamine. Cancer Res 46: 1120-1123, 1986.

29 Golding JP, Wardhaugh T, Patrick L, Turner M, Phillips JB, Bruce JI and Kimani SG: Targeting tumour energy metabolism 
potentiates the cytotoxicity of 5-aminolevulinic acid photodynamic therapy. Br J Cancer 109: 976-982, 2013.

30 Cioli V, Bellocci B, Putzolu S, Malorni W and Demartino C: Anti-spermogenic activity of lonidamine (af-1890) in rabbit. Ultramicroscopy 5: 418-418, 1980.

31 Floridi A, Paggi MG, Datri S, Demartino C, Marcante ML, Silvestrini B and Caputo A: Effect of lonidamine on the energy metabolism of Ehrlich ascites tumor cells. Cancer Res 41: 46614666, 1981.

32 Floridi A, Paggi MG, Marcante ML, Silvestrini B, Caputo A and Demartino C: Lonidamine, a selective inhibitor of aerobic glycolysis of murine tumor cells. J Nat Cancer Inst 66: 497-499, 1981.

33 Floridi A and Lehninger AL: Action of the anti-tumor and antispermogenic agent lonidamine on electron-transport in Ehrlich ascites tumor mitochondria. Arch Biochem Biophys 226: 73-83, 1983.

34 Floridi A, D'Attri S, Paggi MG, Mercant ML, De Martino C, Silvestrini B, Caputo A and Lehninger AL: Binding of lonidamine to Ehrlich ascites tumor and liver mitochondria. In: Membranes in Tumor Growth (TGe a ed.). Amsterdam: Elsevier Biomedical Press, pp. 559-565, 1982

35 Ben-Horin H, Tassini M, Vivi A, Navon G and Kaplan O: Mechanism of action of the antineoplastic drug lonidamine: ${ }^{31} \mathrm{P}$ and ${ }^{13} \mathrm{C}$ nuclear magnetic resonance studies. Cancer Res 55(13): 2814-2821, 1995.

36 Ben-Yoseph O, Lyons JC, Song CW and Ross BD: Mechanism of action of lonidamine in the 9L brain tumor model involves inhibition of lactate efflux and intracellular acidification. J Neurooncol 36: 149-157, 1998.

37 Mardor Y, Kaplan O, Sterin M, Ruiz-Cabello J, Ash E, Roth Y, Ringel I and Cohen JS: Noninvasive real-time monitoring of intracellular cancer cell metabolism and response to lonidamine treatment using diffusion weighted proton magnetic resonance spectroscopy. Cancer Res 60: 5179-5186, 2000.

38 Nancolas B, Guo LL, Zhou R, Nath K, Nelson DS, Leeper DB, Blair IA, Glickson JD and Halestrap AP: The anti-tumour agent lonidamine is a potent inhibitor of the mitochondrial pyruvate carrier and plasma membrane monocarboxylate transporters. Biochem J 473: 929-936, 2016.

39 Guo LL, Shestov AA, Worth AJ, Nath K, Nelson DS, Leeper DB, Glickson JD and Blair IA: Inhibition of mitochondrial complex II by the anticancer agent lonidamine. J Biol Chem 291: 42-57, 2016.

40 Polavarapu A, Stillabower JA, Stubblefield SGW, Taylor WM and Baik MH: The mechanism of guanine alkylation by nitrogen mustards: A Computational study. J Org Chem 77: 5914-5921, 2012.

41 Bhattacharyya PK and Medhi C: Theoretical study on the mechanism of alkylation at $\mathrm{N}-7$ of guanine by few nitrogen mustards. Indian J Chem 44: 1319-1323, 2005.

42 Ekanayake KS and Lebreton PR: Model transition states for methane diazonium ion methylation of guanine runs in oligomeric DNA. J Comp Chem 28: 2352-2365, 2007.

43 Hamza A, Broch H and Vasilescu D: Quantum molecular modeling of the interaction between guanine and alkylating agents-2-nitrogen mustard. J Biomol Struct Dynamics 13: 915924, 1996.

44 Kohn KW, Hartley JA and Mattes WB: Mechanisms of DNAsequence selective alkylation of guanine-N7 positions by nitrogen mustards. Nucleic Acids Res 15: 10531-10549, 1987.
45 Kohn KW, Hartley JA and Mattes WB: Mechanisms of DNA sequence selective alkylation of guanine- $\mathrm{N}^{7}$ positions by nitrogen mustards. Biochem Pharmac 37: 1799-1800, 1988.

46 Shukla PK, Mishra PC and Suhai S: Reactions of DNA bases with the anti-cancer nitrogen mustard mechlorethamine: A quantum chemical study. Chem Physics Lett 449: 323-328, 2007.

47 Canter RJ, Zhou R, Kesmodel SB, Zhang YW, Heitjan DF, Glickson JD, Leeper DB and Fraker DL: Metaiodobenzylguanidine and hyperglycemia augment tumor response to isolated limb perfusion in a rodent model of human melanoma. Ann Surg Oncol 11: 265-273, 2004.

48 Turley RS, Raymond AK and Tyler DS: Regional treatment strategies for in-transit melanoma metastasis. Surg Oncol Clin N Am 20: 79-103, 2011.

49 McQuade JL, Posada LP, Lecagoonporn S, Cain S, Bassett RL, Patel SP, Hwu WJ, Hwu P, Davies MA, Bedikian AY and Amaria RN: A phase I study of TPI 287 in combination with temozolomide for patients with metastatic melanoma. Melanoma Res 26: 604-608, 2016.

50 Trinh VA: Current management of metastatic melanoma. Am J Health-System Pharm 65: S3-S9, 2008.

51 Friedman HS, Kerby $\mathrm{T}$ and Calvert $\mathrm{H}$ : Temozolomide and treatment of malignant glioma. Clin Cancer Res 6: 2585-2597, 2000.

52 Stupp R, Hegi ME, Gilbert MR and Chakravarti A: Chemoradiotherapy in malignant glioma: Standard of care and future directions. J Clin Oncol 25: 4127-4136, 2007.

53 Villano JL, Seery TE and Bressler LR: Temozolomide in malignant gliomas: Current use and future targets. Cancer Chemother Pharmacol 64: 647-655, 2009.

54 Wick W, Platten M and Weller M: New (alternative) temozolomide regimens for the treatment of glioma. Neuro Oncol 11: 69-79, 2009.

55 Adjalle R, Plouin PF, Pacak K and Lehnert H: Treatment of malignant pheochromocytoma. Hormone Metab Res 41: 687-696, 2009.

56 Fazio N, Spada F and Giovannini M: Chemotherapy in gastroenteropancreatic (GEP) neuroendocrine carcinomas (NEC): A critical view. Cancer Treat Rev 39: 270-274, 2013.

57 Kulke MH, Bendell J, Kvols L, Picus J, Pommier R and Yao J: Evolving Diagnostic and treatment strategies for pancreatic neuroendocrine tumors. J Hematol Oncol 4: 29, 2011.

58 McCormack AI, Wass JAH and Grossman AB: Aggressive pituitary tumours: The role of temozolomide and the assessment of MGMT status. Eur J Clin Invest 41: 1133-1148, 2011.

59 Vilar E, Salazar R, Perez-Garcia J, Cortes J, Oberg K and Tabernero J: Chemotherapy and role of the proliferation marker Ki-67 in digestive neuroendocrine tumors. Endocr Relat Cancer 14: 221-232, 2007.

60 Vaughan K and Stevens MFG: Monoalkyltriazenes. Chem Soc Rev 7: 377-397, 1978.

61 Middlemas DS, Stewart CF, Kirstein MN, Poquette C, Friedman HS, Houghton PJ and Brent TP: Biochemical correlates of temozolomide sensitivity in pediatric solid tumor xenograft models. Clin Cancer Res 6: 998-1007, 2000.

Received May 10, 2017

Revised May 24, 2017

Accepted May 25, 2017 